\section{Innocuousness of conjunctival vaccination with Brucella melitensis strain Rev.1 in pregnant Iranian fat-tailed ewes}

\author{
Saeed Alamian, Ramin Bagheri Nejad, \\ Hamid Reza Jalali, Armin Kalantari, \\ Afshar Etemadi
}

Brucellosis Department, Razi Vaccine and Serum Research Institute, Karaj, Iran

\section{Abstract}

Brucella melitensis strain Rev.1 is the most effective vaccine against brucellosis in sheep and goats. In Iran, mass vaccination is carried out all over the country in which adult animals are immunized by subcutaneous injection of reduced doses of the vaccine. However, due to antibody responses elicited by vaccination, concomitant implementation of test-andslaughter is impossible. To overcome the problem, vaccination through conjunctival route is recommended. In this study, serological responses of six pregnant Iranian fat-tailed ewes to conjunctival vaccination with standard doses of the vaccine were evaluated using modified Rose Bengal test, serum agglutination test and indirect ELISA. Besides, vaccine strain excretion in milk and vaginal discharges was also examined by microbiological culture of milk and vaginal swab samples taken one day post-parturition. Animals were vaccinated during the second half of gestation. As the results, antibody titers of five (83.3\%) ewes decreased to the levels not detectable by the tests within three months after vaccination. No vaccine-induced abortions occurred and vaccinated ewes delivered healthy lambs $50.33 \pm 15.56$ (mean \pm standard deviation) days post-vaccination. Vaccine strain was not isolated from milk and vaginal swab samples. Generally, our study shows full doses of $B$. melitensis strain Rev.1 can be used conjunctively to vaccinate pregnant Iranian sheep during late pregnancy without abortifacient effects, prolonged antibody responses and vaccine strain excretion in milk and vaginal discharges. Nevertheless, further studies are required to determine safety and immunogenicity of the vaccine in field conditions.

\section{Introduction}

Brucellosis in sheep and goats is an important zoonotic disease caused mainly by
Brucella melitensis. 1,2 Vaccination of the host animals with $B$. melitensis strain Rev.1 is used worldwide for disease control which has been proved to be the most effective vaccine..$^{3,4}$ It is recommended to immunize replacement animals from 3 to 6 months of age with standard doses of vaccine containing at least $10^{9}$ live cells. ${ }^{5}$ However, there is evidence that effective control of the disease in countries with high prevalence requires immunization of all susceptible young and adult animals in a mass vaccination campaign which is considered as the most practical measure. . $^{6}$

One problem with vaccination of adult animals is antibody responses induced by the vaccine which may last for a long time and cause sero-positivity of vaccinated animals in routine serological tests interfering with detection of infected ones.4,9,10 This makes simultaneous implementation of vaccination and testand-slaughter impossible since vaccinated animals are falsely diagnosed as infected. ${ }^{4}$ Moreover, vaccine-induced abortion and vaccine strain excretion in milk and vaginal discharges may occur.5,10 Vaccination of flocks through conjunctival route is known as one way to solve these problems. $6,10,11$

Small ruminant brucellosis is an enzootic disease in Iran causing abortion in different parts of the country. 12 Mass vaccination has been the main control measure since 2003 in which adult animals are vaccinated subcutaneously using reduced doses of the vaccine. ${ }^{12}$ Nevertheless, there are field reports showing long-lasting sero-positivity of vaccinated adult sheep and goats and abortions in pregnant animals attributed to the vaccination. Therefore, this study was done to evaluate serological responses of pregnant fat-tailed ewes to ocular vaccination with standard doses of $B$. melitensis strain Rev.1 as well as its safety in terms of abortion induction and vaccine strain excretion in milk and vaginal secretions.

\section{Materials and Methods}

\section{Animals and vaccination}

Eleven pregnant Iranian fat-tailed ewes were randomly selected from a known brucellosis-free flock. Selected animals were negative in modified Rose Bengal test (mRBT), serum agglutination test (SAT) and indirect ELISA (iELISA) carried out twice with a month's interval. Six ewes were vaccinated during third to fifth month of pregnancy with conjunctival $B$. melitensis strain Rev.1 vaccine containing $10^{9}$ colony forming units (CFU) per dose. Other ewes were used as controls in which normal saline was used instead of vaccine at the same time. The vaccine used in the study was produced in Razi Vaccine and Serum
Correspondence: Ramin Bagheri Nejad, Brucellosis Department, Razi Vaccine and Serum Research Institute, 3197619751 Karaj, P.0. Box: 31975/148 Iran.

Tel.: +98.263.4570038 - Fax: +98.263.4552194.

E-mail: r.bagherinejad@rvsri.ac.ir

Key words: Brucellosis; Vaccination; Sheep; Brucella melitensis strain Rev.1; Conjunctival vaccine.

Acknowledgements: The authors would like to thank Mr. Ali Asghar Izadyar, Mr. Mohammad Saffari and other personnel of Brucellosis Department at Razi Vaccine and Serum Research Institute for their kind technical assistance. The study was funded by Razi Vaccine and Serum Research Institute.

Contributions: RBN designed the study. SA, RBN, HRJ, AK and AE performed the study. RBN did the statistical analysis. RBN prepared the draft which was approved by all authors.

Conflict of interest: the authors declare no potential conflict of interest.

Received for publication: 8 May 2015.

Accepted for publication: 24 June 2015.

This work is licensed under a Creative Commons Attribution NonCommercial 3.0 License (CC BYNC 3.0).

(C) Copyright S. Alamian et al., 2015

Licensee PAGEPress srl, Italy

Veterinary Science Development 2015; 5:5997

doi:10.4081/vsd.2015.5997

Research Institute according to standard procedures.5,13 The original seed for vaccine production was obtained from Animal Health and Veterinary Laboratories Agency (AHVLA), Weybridge, UK. Vaccinated and control animals were kept separately in the same conditions.

\section{Serological evaluation}

Animals were bled every 2 weeks for three months after immunization to evaluate serological responses to vaccination. Serum samples were examined using mRBT, SAT and iELISA. RBT and SAT antigens were produced in Razi Vaccine and Serum Research Institute based on standard methods,, 13 as described previously. ${ }^{14}$ For mRBT, one drop of the antigen was mixed with three drops of the serum sample. ${ }^{15}$ Indirect ELISA was performed using PrioCHECK ${ }^{\circledR}$ Brucella Ab (Prionics AG, Schlieren, Switzerland) according to the manufacturer's instructions.

\section{Bacteriological examination}

To determine vaccine strain excretion in 
milk and vaginal discharges, milk and vaginal swab samples were taken within 24 hours after abortion or parturition. Milk samples were first centrifuged at 6000-7000 rpm for 15 minutes and then supernatant cream and precipitated pellet were cultured. ${ }^{13}$ Swab samples were cultured directly on solid media. Brucella agar medium (BD, USA) was used for vaccine strain isolation which was supplemented with Brucella selective antibiotics (Oxoid, Basingstoke, UK) and 5\% (v/v) horse serum following manufacturer's instructions. For each sample, at least 3 plates were inoculated.

\section{Results}

\section{Serological responses after vaccination}

While control ewes remained negative during the study, vaccinated animals showed antibody responses from the second week after immunization. Percents of positive vaccinated ewes in mRBT and iELISA at two-week intervals over the study period are presented in Figure 1. Two weeks post-vaccination, all animals were positive in mRBT but none of them in iELISA. Five ewes (83.3\%) showed positive results in iELISA after four weeks. The mRBT and iELISA results of each ewe were similar from fourth week on. After 12 weeks, only one vaccinated ewe (16.7\%) remained positive detected by the two tests.

Evaluation of antibody titers using SAT demonstrated a falling trend over time (Figure 2). All animals showed increased antibody titers after two weeks which declined gradually afterwards in a way that 5 ewes (83.3\%) had no SAT titers twelve weeks after vaccine inoculation. The only ewe, which had antibody titers detectable by SAT, was also reactive in mRBT and iELISA.

\section{Vaccine safety}

No abortion occurred following vaccination and all vaccinated animals had normal delivery with healthy lambs $50.33 \pm 15.56$ (mean \pm SD) days after vaccine inoculation. $B$. melitensis strain Rev.1 was not isolated by microbiological methods from milk and vaginal discharges of vaccinated sheep within 24 hours postpartum.

\section{Discussion and Conclusions}

In Iran, brucellosis in small ruminants is an important enzootic disease which is a public health burden. Nomadic raising of sheep and goats, traditional production practices, illegal animal imports and uncontrolled movements of flocks within the country contribute to the difficulties in disease control. In these conditions, control of the disease has been mainly based on mass vaccination of young and adult animals using subcutaneous administration of full and reduced doses of $B$. melitensis strain Rev.1, respectively. ${ }^{12}$ However, the disease still remains prevalent in different parts of the country diagnosed as a significant cause of abortion in sheep and goats. ${ }^{12}$

Although we previously demonstrated that reduced doses containing less than or equal to $10^{6}$ bacteria can be safely used to immunize pregnant ewes with short-lasting serological responses, ${ }^{14}$ field reports show persistence antibody responses and abortions caused by vaccination. It is known that the innocuousness of the vaccine in adult and pregnant animals depends on vaccine dose, time of vaccination during gestation and administration route. ${ }^{8,16}$ Hence, these observations may be partly due to the fact that according to a standard approved by National Brucellosis Expert Committee, the reduced dose of vaccine used for adult animals immunization can contain up to $4 \times 10^{6}$ colony forming units (CFU) per dose. In addition, subcutaneous use of vaccine and extended lambing season in Iran, which results in presence of pregnant animals throughout the year in flocks, could be influential. Therefore, ocular inoculation of the vaccine is considered as an alternative proved to be safer. $6,8,10,16$

In our experiment, all animals were detected as positive using mRBT two weeks after immunization. Stournara et al. ${ }^{9}$ also reported a hundred percent positive results in mRBT of non-pregnant ewes 21 days post-vaccination. In another study by Zundel et al.,16 all ewes vaccinated at mid-pregnancy using the same dose as ours were positive in RBT 2 weeks following vaccination.

The proportion of positive vaccinated ewes in iELISA reached its maximum after 4 weeks. A similar result has been observed by Stournara et al. ${ }^{9}$ which was attributed to the higher affinity of the conjugate used in the assay to immunoglobulin G (IgG). It has also been reported that more than 70 percent of non-vaccinated ewes were detected as negative by iELISA 14 days after challenge with the virulent strain during pregnancy. ${ }^{17}$ Because the ewes used in our study were from a brucellosis-free flock without previous exposure to the pathogen, and according to the explanation provided by Stournara et al., ${ }^{9}$ negative iELISA results of these naive ewes two weeks after vaccine inoculation suggest antibody responses may be mainly of IgM class at this time. The percentage of positive animals detected by iELISA and mRBT decreased rapidly from 6 weeks post-vaccination to the end of study. Similar performance of iELISA and mRBT in our study is in agreement with results of the study carried out by Stournara et al. ${ }^{9}$ However, in the latter study $72.6 \%$ and $84 \%$ of animals remained positive in iELISA and mRBT, respectively 91 days after immunization, but in our study only $16.7 \%$ were positive in both tests 12 weeks post-vaccination. This differ-

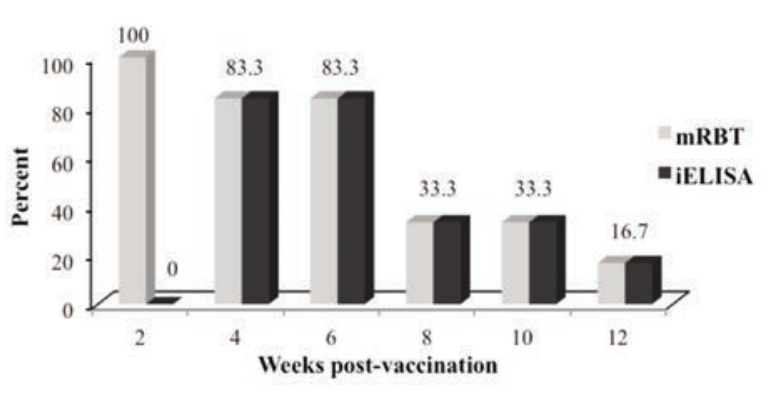

Figure 1. Percent of positive vaccinated ewes in modified Rose Bengal test and indirect ELISA.

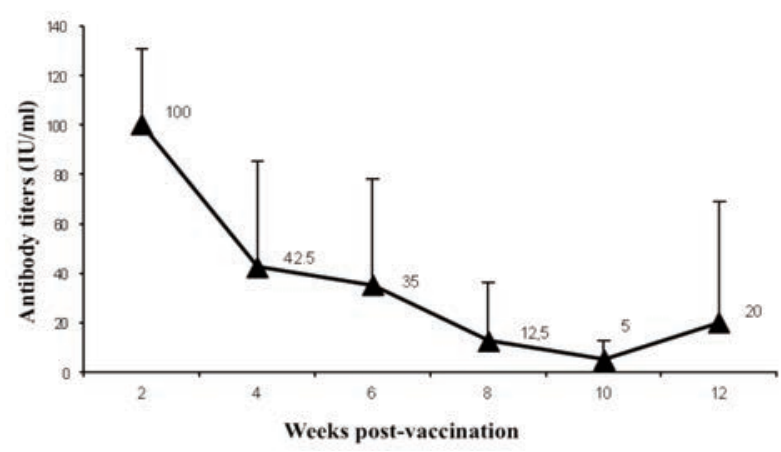

Figure 2. Mean \pm standard deviation of serum agglutination titers in vaccinated animals. 
ence may be due to age and physiologic status of animals when vaccinated or breed variation in antibody responses. 7,18

Evaluation of serological responses during the study period using SAT revealed antibody titers fell from the peak reached at week two toward the end of study. SAT results are compatible with the other two tests and the only ewe, which had antibody titer of diagnostic value 12 week after immunization, was also detected as positive in iELISA and mRBT at this interval. While serum antibody level of this ewe was decreasing from $120 \mathrm{IU} / \mathrm{mL}$ six weeks following vaccination to $15 \mathrm{IU} / \mathrm{mL}$ at week 10 , there was a further surge in its antibody titer $(120 \mathrm{IU} / \mathrm{mL})$ two weeks later at $12^{\text {th }}$ week. For this animal, parturition occurred 66 days post-immunization 4 days prior to blood collection for the $10^{\text {th }}$ week. This suggests that parturition might have effects on antibody responses to vaccination.

Use of vaccine through conjunctival route during second half of pregnancy was safe in terms of abortion induction, and no vaccine excretion in milk and fetal materials was detected soon after delivery. Rev.1 strain delivered conjunctivally is known to have a spread confined mainly to head lymph nodes. ${ }^{8}$ Considering normal delivery of all animals one to two months following vaccine inoculation and as vaccine strain was not isolated immediately after parturition, it seems Rev.1 strain was not generalized to the uterus and mammary gland. Although there is no a completely safe way to use Rev.1 vaccine in pregnant small ruminants, ${ }^{4}$ it is known that conjunctival administration of the vaccine during late pregnancy or before breeding can reduce risks of vaccine-induced abortions and vaccine strain excretion in milk and vaginal discharges, ${ }^{5}$ which was also proved in Iranian fat-tailed ewes in this study.

In general, the present experiment showed serological responses to ocular vaccination of pregnant Iranian fat-tailed sheep with standard doses of $B$. melitensis strain Rev.1 disappeared in a considerable proportion of animals within 12 weeks. Moreover, use of vaccine during late pregnancy did not cause vaccination- induced abortion and Rev.1 strain excretion in milk and vaginal discharges during immediate postpartum period. Nevertheless, further investigations are required to assess vaccine performance in field conditions.

\section{References}

1. Diaz Aparicio E. Epidemiology of brucellosis in domestic animals caused by Brucella melitensis, Brucella suis and Brucella abortus. Rev Sci Tech 2013;32:4351, 3-60.

2. Garin-Bastuji B, Blasco JM, Grayon M, Verger JM. Brucella melitensis infection in sheep: present and future. Vet Res 1998;29:255-74.

3. Schurig GG, Sriranganathan N, Corbel MJ. Brucellosis vaccines: past, present and future. Vet Microbiol 2002;90:479-96.

4. Olsen SC, Stoffregen WS. Essential role of vaccines in brucellosis control and eradication programs for livestock. Expert Rev Vaccines 2005;4:915-28.

5. OIE. Manual of diagnostic tests and vaccines for terrestrial animals. Paris: World Organization for Animal Health; 2009.

6. Blasco JM. Control and eradication strategies for Brucella melitensis infection in sheep and goats. Prilozi 2010;31:145-65.

7. Nicoletti P. Brucellosis: past, present and future. Prilozi 2010;31:21-32.

8. Jimenez de Bagues MP, Marin CM, Barberan M, Blasco JM. Responses of ewes to B. melitensis Revl vaccine administered by subcutaneous or conjunctival routes at different stages of pregnancy. Ann Vet Res 1989;20:205-13.

9. Stournara A, Minas A, BourtziChatzopoulou E, et al. Assessment of serological response of young and adult sheep to conjunctival vaccination with Rev-1 vaccine by fluorescence polarization assay (FPA) and other serological tests for B. melitensis. Vet Microbiol 2007;119:53-64.

10. Blasco JM. A review of the use of $B$. melitensis Rev 1 vaccine in adult sheep and goats. Prev Vet Med 1997;31:275-83.

11. Fensterbank R, Verger JM, Grayon M. Conjunctival vaccination of young goats with Brucella melitensis strain Rev 1. Ann Vet Res 1987;18:397-403.

12. Behroozikhah AM, Bagheri Nejad R, Amiri $\mathrm{K}$, Bahonar AR. Identification at biovar level of Brucella isolates causing abortion in small ruminants of iran. $\mathrm{J}$ Pathogens 2012;2012:357235.

13. Alton GG, Jones LM, Angus RD, Verger JM. Techniques for the Brucellosis Laboratory. Paris: Institut National de la Recherche Agronomique; 1988.

14. Ebrahimi M, Nejad RB, Alamian S, et al. Safety and efficacy of reduced doses of Brucella melitensis strain Rev. 1 vaccine in pregnant Iranian fat-tailed ewes. Vet Ital 2012;48:405-12.

15. Ferreira AC, Cardoso R, Travassos Dias I, et al. Evaluation of a modified Rose Bengal test and an indirect enzyme-linked immunosorbent assay for the diagnosis of Brucella melitensis infection in sheep. Vet Res 2003;34:297-305.

16. Zundel E, Verger JM, Grayon M, Michel R. Conjunctival vaccination of pregnant ewes and goats with Brucella melitensis Rev 1 vaccine: safety and serological responses. Ann Vet Res 1992;23:177-88.

17. Duran-Ferrer M, Leon L, Nielsen K, et al. Antibody response and antigen-specific gamma-interferon profiles of vaccinated and unvaccinated pregnant sheep experimentally infected with Brucella melitensis. Vet Microbiol 2004;100:219-31.

18. Jimenez de Bagues MP, Marin CM, Blasco $\mathrm{JM}$, et al. An ELISA with Brucella lipopolysaccharide antigen for the diagnosis of B. melitensis infection in sheep and for the evaluation of serological responses following subcutaneous or conjunctival B. melitensis strain Rev 1 vaccination. Vet Microbiol 1992;30:233-41. 